\title{
Article
}

\section{A new species of Conidiobolus with chlamydospores from Dabie Mountains, Eastern China}

\author{
Nie $Y^{1,2}$, Tang $X X^{1}$, Liu $X Y^{3^{*}}$ and Huang $B^{1 *}$ \\ ${ }^{1}$ Anhui Provincial Key Laboratory for Microbial Pest Control, Anhui Agricultural University, Hefei 230036, China \\ ${ }^{2}$ School of Civil Engineering and Architecture, Anhui University of Technology, Ma anshan 243002, China \\ ${ }^{3}$ State Key Laboratory of Mycology, Institute of Microbiology, Chinese Academy of Sciences, Beijing 100101, China
}

Nie Y, Tang XX, Liu XY, Huang B 2017 - A new species of Conidiobolus with chlamydospores from Dabie Mountains, Eastern China. Mycosphere 8(7), 809-816, Doi 10.5943/mycosphere/8/7/1

\begin{abstract}
Conidiobolus is a widely distributed genus throughout the world, and its resting spores include zygospores, chlamydospores and villose conidia. Conidiobolus dabieshanensis is confirmed as a new species with chlamydospores within the genus Conidiobolus based on the morphological evidence and the molecular data of LSU rDNA region. It is morphologically allied with $C$. adiaeretus and $C$. firmipilleus and it is phylogenetically closely related to C. humicolus, C. chlamydosporus and $C$. firmipilleus. The new species differs from $C$. adiaeretus by the absence of capilliconidia, from $C$. firmipilleus and $C$. chlamydosporus by its size of primary conidia and slender and curving germ hyphae arising from a single conidium, and from $C$. humicolus by its larger primary conidia and the absence of zygospores.
\end{abstract}

Key words - Entomophthorales - Morphology - Molecular phylogeny

\section{Introduction}

The genus Conidiobolus Bref. (Ancylistaceae, Entomophthorales) typified by C. utriculosus Bref. was originally isolated from basidiomycete fruit bodies (Brefeld 1884). It is widely distributed in soils and decaying leaves and sometimes isolated from keratin, living ferns and rotten vegetables (Kwon-Chung 2012, Nie et al. 2012). In addition, C. coronatus, $C$. incongruous, and $C$. lamprauges have been reported as pathogens of humans and animals (Vilela et al. 2010, Mendoza et al. 2014).

A total of 34 species are currently recognized within Conidiobolus (Huang et al. 2007; King 1976a, b, 1977; Nie et al. 2012, 2016). Three subgenera Delacroixia, Capillidium and Conidiobolus were introduced based on the types of secondary conidia (Ben-Ze'ev \& Kenneth 1982), and followed by Humber $(1989,1997)$. Recent multigene phylogenetic analyses revealed at least 3 lineages in the genus Conidiobolus, but the subgeneric boundaries of Ben-Ze'ev \& Kenneth are not supported (Gryganskyi et al. 2013).

Three types of resting spores are presented in Conidiobolus species. They are zygospores, chlamydospores and villose conidia. The zygospores are the most common, which are double walled and formed inside the larger one of the two conjugating cells (Humber 1981, 1989). Within Conidiobolus, 22 species produce zygospores. Among the remaining species, only C. coronatus produces villose conidia, and $C$. adiaeretus, $C$. eurymitus, $C$. firmipilleus, $C$. humicolus, $C$. lachnodes, $C$. pumilus, and $C$. rhysosporus form chlamydospores with a single thickened wall layer 
(King 1977). The present study introduces a new species of Conidiobolus with chlamydospores based on morphological features and phylogenetic data.

\section{Materials \& Methods}

\section{Sample collection, strain isolation and morphological identification}

Soil samples were collected from Dabie Mountains, Huoshan County, Anhui Province, Eastern China. The canopy plating approach in this study followed Drechsler (1952) and King (1976a). In the laboratory, a Petri dish containing potato dextrose agar (PDA, Difco) was inverted over the soil, incubated at $21^{\circ} \mathrm{C}$ and examined daily. When fungi were detected on the PDA canopy, discharged conidia transferred to a new PDA plate for morphological study. The measurements of different fungal structures were taken as described by King (1976a). The specimen has been deposited in the Research Center for Entomogenous Fungi of Anhui Agricultural University (RCEF) and duplicated in the China General Microbiological Culture Collection Center (CGMCC). The dried cultures were deposited in RCEF and the Herbarium Mycologicum Academiae Sinicae (HMAS).

\section{DNA extraction, PCR and sequencing}

After incubation on PDA at $21^{\circ} \mathrm{C}$ for seven days, total genomic DNA was extracted from the mycelium scraped from the cellophane with modified CTAB method (Watanabe et al. 2010), and stored at $-20^{\circ} \mathrm{C}$. The LSU rDNA region was amplified with the primers LROR (5'ACCCGCTGAACTTAAGC-3') and LR5 (5'-TCCTGAGGGAAACTTCG-3') (Vilgalys et al. 1990), and the PCR reaction used in this study has been described by Liu (Liu et al. 2005). PCR products were sequenced by Shanghai Genecore Biotechnologies Company (Shanghai, China) with the same primers. Accession numbers for sequences used for phylogenetic analyses are provided (Table 1).

\section{Phylogenetic analysis}

Referring to King (1976b), more LSU rDNA sequences were downloaded from GenBank. Batkoa apiculata, Entomophthora muscae, and Erynia conica were retrieved as outgroups. Multiple sequences were aligned with BioEdit (Hall 1999) and Clustal X (Thompson et al. 1997), and the alignment was deposited to TreeBASE (Submission ID: 20453; Study Accession URL: http://purl.org/phylo/treebase/phylows/study/TB2:S20453). Maximum Parsimony (MP) and Bayesian Inference (BI) were applied to the LSU rDNA dataset. All characters were weighted, and gaps were treated as missing data. PAUP* 4.0b10 (Swofford 2002) was used to perform the MP analysis. Branch swapping algorithm was tree bisection-reconnection (TBR) and MulTrees were used. Branch support was estimated with bootstrapping by using 1,000 replicates (Felsenstein 1985). Modeltest 3.7 (Posada \& Crandall 1998) was used to determine the best-fit evolution mode for BI. The BI was implemented by Markov Chain Monte Carlo (MCMC) sampling within the software MrBayes 3.1.2 (Ronquist \& Huelsenbeck 2003). The MCMC chains ran until a critical value for the topological convergence diagnostic was less than 0.005 .

\section{Results}

\section{Phylogenetic analyses}

The final partial LSU sequence alignment includes 39 strains, and consists of 1025 characters, of which 604 characters are parsimony-informative and 112 characters are parsimony-uninformative. The heuristic search using maximum parsimony (MP) generated three parsimonious trees $(\mathrm{TL}=$ $3378, \mathrm{CI}=0.4307, \mathrm{HI}=0.5693, \mathrm{RI}=0.7035, \mathrm{RC}=0.3030$ ), one of which is selected and shown in Figure 1. The GTR+I+G nucleotide substitution model is used based on the Akaike Information Criterion (AIC). One tree is saved to a file within every 100 generations for a total of 1,000,000 MCMC generations, and the average standard deviation of split frequencies in Bayesian Inference is 0.004833 , below 0.005 . Three clades present on the phylogenetic tree, and $C$. dabieshanensis grouped in Clade I, being most closely related to $C$. humicolus, $C$. firmipilleus and $C$. chlamydosporus. 
Table 1 The species in this study and their GenBank accession numbers of LSU rDNA*.

\begin{tabular}{|c|c|c|}
\hline Species & Strains & GeneBank Number \\
\hline Conidiobolus adiaeretus & ARSEF $451(\mathrm{~T})$ & KC461182 \\
\hline C. antarcticus & ARSEF 6913 (T) & DQ364207 \\
\hline C. bangalorensis & ARSEF 449 (T) & DQ364204 \\
\hline C. brefeldianus & ARSEF $452(\mathrm{~T})$ & EF392382 \\
\hline C. chlamydosporus & ATCC $12242(\mathrm{~T})$ & JF816212 \\
\hline C. coronatus & NRRL 28638 & AY546691 \\
\hline C. coronatus & ARSEF 525 & DQ364205 \\
\hline C. coronatus & RCEF 4518 & JN131537 \\
\hline C. couchii & ATCC $18152(\mathrm{~T})$ & JN131538 \\
\hline C. dabieshanensis & CGMCC $3.15763(\mathrm{~T})$ & KY398125 \\
\hline C. firmipilleus & ARSEF 6384 & JX242592 \\
\hline C. gonimodes & ATCC 14445 (T) & JF816221 \\
\hline C. heterosporus & RCEF 4430 & JF816225 \\
\hline C. humicolus & ATCC 28849 (T) & JF816220 \\
\hline C. iuxtagenitus & ARSEF 6378 (T) & KC788410 \\
\hline C. iuxtagenitus & RCEF 4445 & JX946695 \\
\hline C. khandalensis & ATCC $15162(\mathrm{~T})$ & KX686994 \\
\hline C. lachnodes & ARSEF 700 & KC788408 \\
\hline C. lichenicolus & ATCC $16200(\mathrm{~T})$ & JF816216 \\
\hline C. lobatus & ATCC 18153 (T) & JF816218 \\
\hline C. marcosporus & ATCC $16578(\mathrm{~T})$ & KY398124 \\
\hline C. mycophilus & ATCC 16199 (T) & KX686995 \\
\hline C. nodosus & ATCC 16577 (T) & JF816217 \\
\hline C. osmodes & ARSEF 79 & EF392371 \\
\hline C. osmodes & RCEF 4447 & JN131539 \\
\hline C. parvus & ATCC 14634 (T) & KX752051 \\
\hline C. paulus & ARSEF $450(\mathrm{~T})$ & KC788409 \\
\hline C. polytocus & ATCC 12244 (T) & JF816213 \\
\hline C. pumilus & ARSEF $453(\mathrm{~T})$ & EF392383 \\
\hline C. rhysosporus & ATCC 12588 (T) & JN131540 \\
\hline C. sinensis & RCEF $4952(\mathrm{~T})$ & JF816224 \\
\hline C. stilbeus & RCEF $5584(\mathrm{~T})$ & KP218522 \\
\hline C. stromoideus & ATCC $15430(\mathrm{~T})$ & JF816219 \\
\hline C. terrestris & ATCC 16198 (T) & KX752050 \\
\hline C. thromboides & ATCC 12587 (T) & JF816214 \\
\hline C. thromboides & RCEF 4492 & JF816223 \\
\hline Batkoa apiculata & ARSEF 3130 & EF392404 \\
\hline Entomophthora muscae & ARSEF 3074 & DQ273772 \\
\hline Erynia conica & ARSEF 1439 & EF392396 \\
\hline
\end{tabular}

*The taxonomy of Conidiobolus refers to the scheme of King (1976b). ARSEF = ARS Entomopathogenic Fungus Collection (Ithaca, U.S.A.). ATCC = American Type Culture Collection (Manassas, U.S.A). CGMCC = China General Microbiological Culture Collection Center (Beijing, China). NRRL = ARS Culture Collection (Peoria, U.S.A). RCEF = Research Center for Entomogenous Fungi (Hefei, China). T = ex-type.

Conidiobolus dabieshanensis Y. Nie \& B. Huang, sp. nov.

Figs $2-13$ Index Fungorum number: IF 552756; Facesoffungi number: FoF 02877 (Jayasiri et al. 2015) Etymology -dabieshanensis (Lat.) = Referring to the region where the fungus was isolated. This species is mainly characterized by the formation of chlamydospores and the slender and curving germ hyphae arising from a single conidium. It differs from $C$. adiaeretus by the absence 


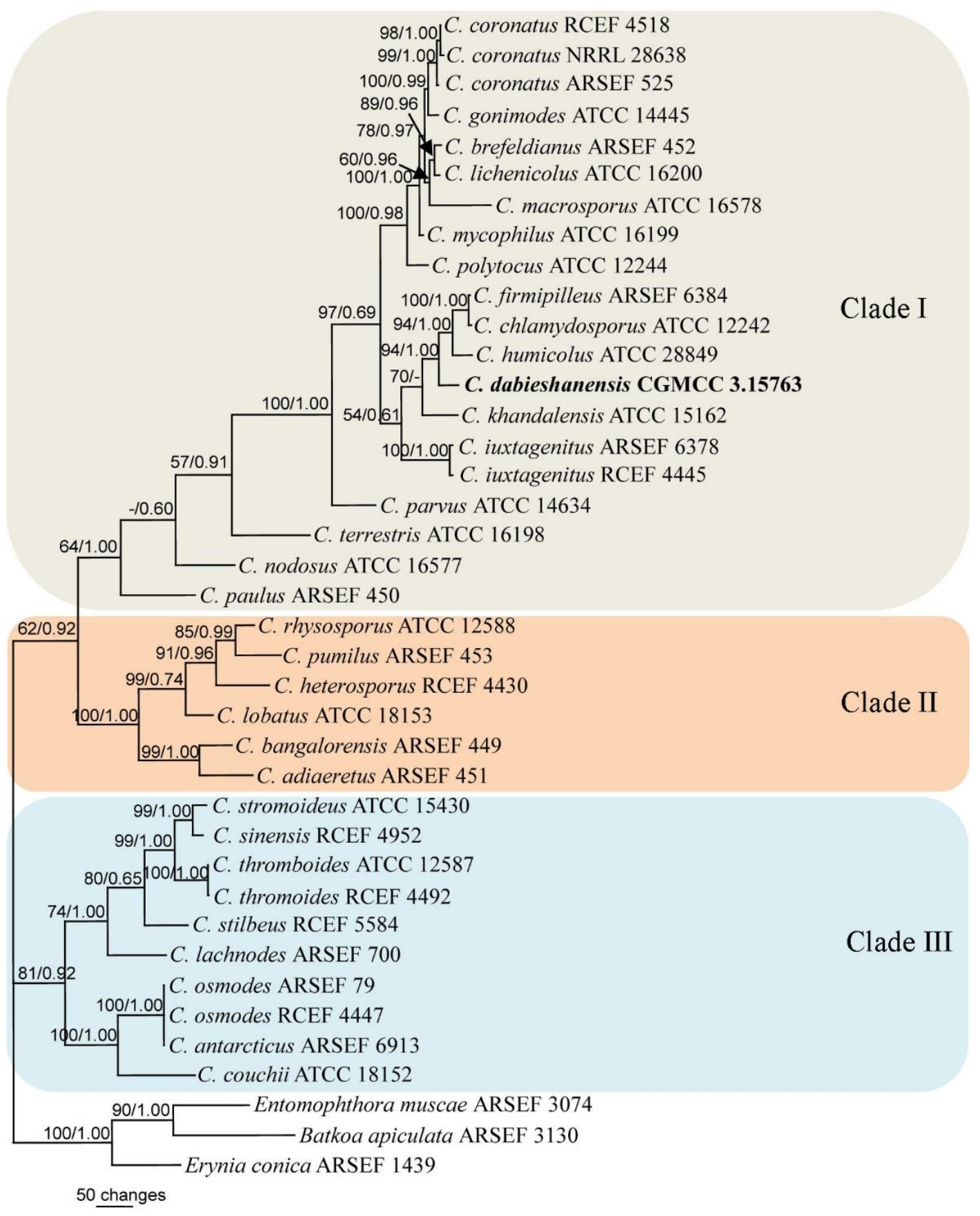

Figure 1 - Phylogenetic tree of Conidiobolus constructed with maximum parsimony analysis of LSU rDNA sequence data, with Entomophthora muscae, Batkoa apiculata and Erynia conica as outgroup taxa. Branches are labeled with maximum parsimony bootstrap value (BP), and Bayesian posterior probabilities (BPP) more than 50\% and 0.60, respectively. The new species of $C$. dabieshanensis is in bold. Bar at the bottom left represents 50 changes in the parsimony analyses.

of capilliconidia, and from $C$. firmipilleus by slightly larger primary conidia and slender and curving germ hyphae. Phylogenetically, it is closely related to $C$. humicolus, $C$. firmipilleus and $C$. chlamydosporus, but differs from $C$. humicolus by its larger primary conidia and the absence of zygospores, and from $C$. chlamydosporus by its slightly smaller primary conidia and curving germ hyphae.

Known distribution - Anhui province, China

Material examined - China, Anhui Province, Huoshan County, Dabie Mountains, $31^{\circ} 18^{\prime} \mathrm{N}$, $116^{\circ} 01^{\prime} \mathrm{E}$, isolated from soil, 12 April 2016, XX Tang, (RCEF6329, Holotype; HMAS255215, Isotype) - ex-type culture CGMCC 3.15763. 

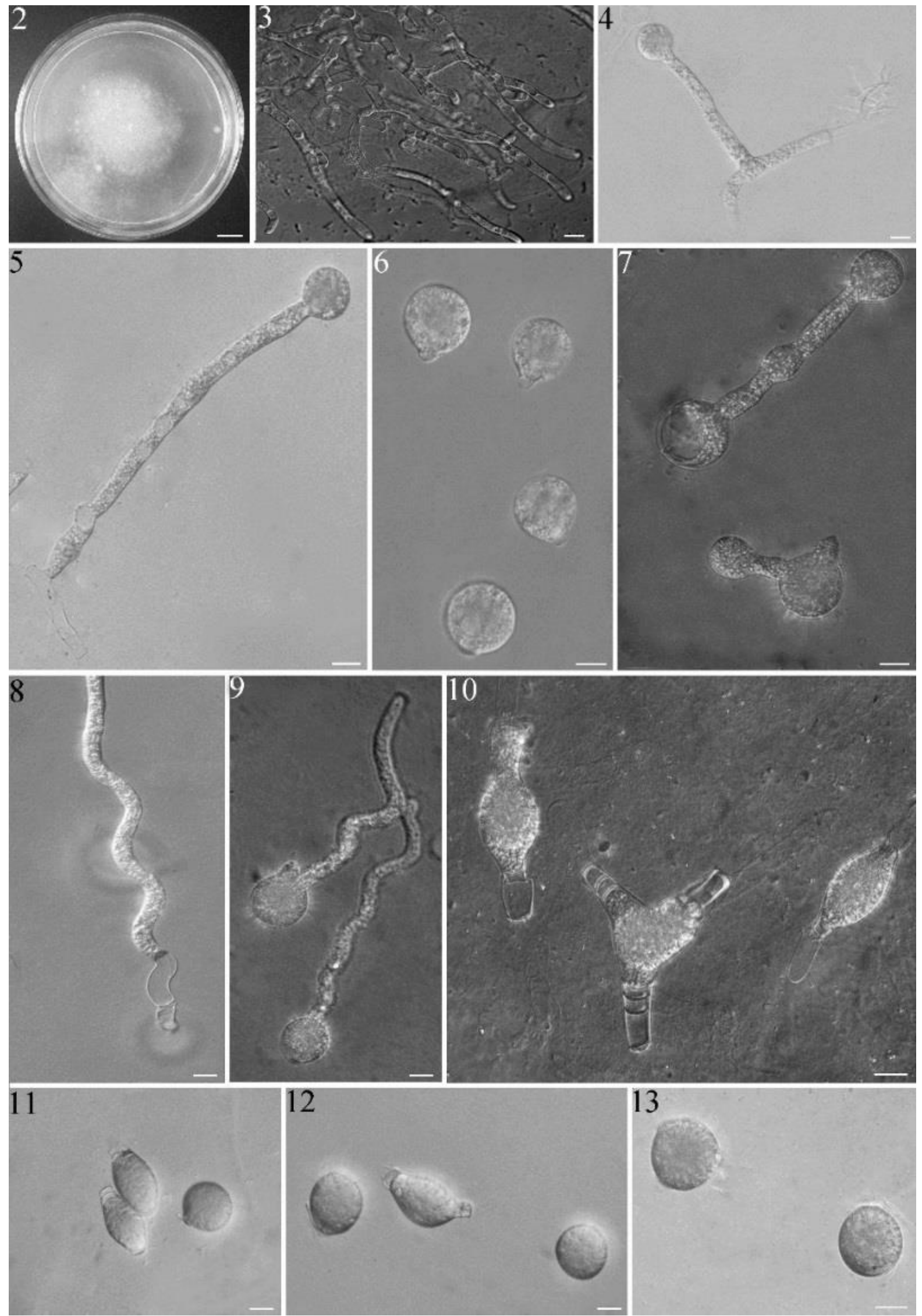

13

Figures 2-13-Conidiobolus dabieshanensis. 2. Colony on PDA after 3 days at $21^{\circ} \mathrm{C}$. 3. Moderately branched at the colony edge. 4, 5. Primary conidiophores arising from mycelia. 6. Primary conidia. 7. Producing secondary conidia. 8, 9. Slender and curving germ hyphae arising from a single conidium. 10. Forming chlamydospores. 11, 12, 13. Chlamydospores. - Bars: $2=10 \mathrm{~mm}, 3-13=20$ $\mu \mathrm{m}$. 
Notes - Colonies on PDA at $21^{\circ} \mathrm{C}$ after 3 days, white, reaching ca $52 \mathrm{~mm}$ in diameter. Mycelia colorless, moderately branched at the colony edge, $8-16 \mu \mathrm{m}$ wide. Primary conidiophores, colorless, unbranched and producing a single globose conidium without widening upward, extending a length of (60-)80-155(-287) $\mu \mathrm{m}$ into the air, 9-17 $\mu \mathrm{m}$ wide. Primary conidia forcibly discharged, colorless, globose to subglobose, measuring 32.5-45 $\mu \mathrm{m}$ in greatest length and 29-38 $\mu \mathrm{m}$ in total width including a basal papilla 4-11 $\mu \mathrm{m}$ high and 9-16 $\mu \mathrm{m}$ wide. After discharging onto $2 \%$ water-agar, similar and smaller secondary conidia arising from primary conidia, slender and curving germ hyphae arising from a single conidium. Chlamydospores formed intercalarily within assimilative hyphae after 3 days, mostly subspherical to elongate ellipsoidal, rarely barrel-shaped, 33-50 $\times 22-35 \mu \mathrm{m}$.

\section{Discussion}

The presence and nature of zygospores or azygospores is a useful ancillary characteristic in Conidiobolus (Humber 1989). Taking into account the types of resting spores in Conidiobolus presented by King (1976a,b, 1977), morphological features were compared among seven Conidiobolus species which form chlamydospores, and the length of primary conidia of the new species $C$. dabieshanensis are mostly related to $C$. adiaeretus $(15-46 \times 13-45 \mu \mathrm{m})$ and $C$. firmipilleus $(8-40 \times 6.5-33 \mu \mathrm{m})$. However, C. dabieshanensis differs from $C$. adiaeretus by the absence of capilliconidia (Drechsler 1953a, Callaghan et al. 2000), and from C. firmipilleus due to the slightly larger primary conidia and curving germ hyphae (Drechsler 1953b, King 1977). As a note, microconidia in $C$. dabieshanensis are not observed.

The sequences of LSU rDNA region have been proved to be effective in distinguishing the Conidiobolus species (Nie et al. 2012, 2016), and the phylogenetic tree (Fig. 1) inferred from this locus demonstrated that 36 Conidiobolus strains (contain 26 ex-types) form three Clades which was almost consistent with the phylogenetic analysis of Conidiobolus based on the datas of nucLSU, nucSSU, RPB2 and mtSSU by Gryganskyi et al. (2013). In the Clade I, C. dabieshanensis forms a well-supported clade with $C$. humicolus, $C$. firmipilleus and $C$. chlamydosporus $(\mathrm{BP}=94, \mathrm{PP}=1.00)$, but morphologically differs from $C$. humicolus $(26-38 \times 23-34 \mu \mathrm{m})$ by its larger primary conidia and the absence of zygospores, and from $C$. chlamydosporus $(18-50 \times 15-45 \mu \mathrm{m})$ by its slightly smaller primary conidia and curving germ hyphae (Drechsler 1955, Srinivasan \& Thirumalachar 1961). The morphological relative $C$. adiaeretus, is phylogenetically distantly related and falls in the Clade II.

Both morphological and phylogenetic evidences support that the specimen isolated from Eastern China is a new species within the genus Conidiobolus.

\section{Acknowledgements}

This project was supported by the National Natural Science Foundation of China (No. 30770008 and 31370068) and the Key Science Research Project of Anhui Province (No. TD200708).

\section{References}

Ben-Ze'ev IS, Kenneth RG. 1982 - Features-criteria of taxonomic value in the Entomophthorales: I. A revision of the Batkoan classification. Mycotaxon 14, 393-455.

Brefeld O. 1884 - Conidiobolus utriculosus und minor. Untersuchungen aus der Gesammtgebiete der Mykologie 6(2), 35-78.

Callaghan AA, Waters SD, Manning RJ. 2000 - Alternative repetitional conidia in Conidiobolus adiaeretus: development and germination. Mycological Research 104, 1270-1275.

Drechsler C. 1952 - Widespread distribution of Delacroixia coronata and other saprophytic Entomophthoraceae in plant detritus. Science 115, 575-576.

Drechsler C. 1953a - Three new species of Conidiobolus isolated from leaf mold. Journal of the Washington Academy of Science 43(2), 29-34.

Drechsler C. 1953b - Two new species of Conidiobolus occurring in leaf mold. American Journal of Botany 40(3), 104-115. 
Drechsler C. 1955 - Two new species of Conidiobolus that produce microconidia. American Journal of Botany 42(9), 793-802.

Felsenstein J. 1985 - Confidence limits on the bootstrap: an approach using the bootstrap. Evolution $38,783-791$.

Gryganskyi AP, Humber RA, Smith ME, Hodge K, Huang B, Voigt K, Vilgalys R. 2013 Phylogenetic lineages in Entomophthoromycota. Persoonia 30, 94-105.

Hall TA. 1999 - Bioedit: a user-friendly biological sequence alignment editor and analysis program for Windows 95/98/NT. Nucleic Acids Symposium Series 41, 95-98.

Huang B, Humber RA, Hodge KT. 2007 - A new species of Conidiobolus from Great Smoky Mountains National Park. Mycotaxon 100, 227-233.

Humber RA. 1981- An alternative view of certain taxonomic criteria used in the Entomophthorales (Zygomycetes). Mycotaxon 13, 191-240.

Humber RA. 1989 - Synopsis of a revised classification for the Entomophthorales (Zygomycotina). Mycotaxon 34, 441-460.

Humber RA. 1997 - Fungi: identification. In: LA Lacey (ed.). Manual of Techniques in Insect Pathology. London, Academic Press.

Jayasiri SC, Hyde KD, Ariyawansa HA, Bhat J et al. 2015 - The faces of fungi database: fungal names linked with morphology, phylogeny and human impacts. Fungal Diversity 74, 3-18.

King DS. 1976a - Systematics of Conidiobolus (Entomophthorales) using numerical taxonomy I. Taxonomic considerations. Canadian Journal of Botany 54, 45-65.

King DS. 1976b-Systematics of Conidiobolus (Entomophthorales) using numerical taxonomy II. Taxonomic considerations. Canadian Journal of Botany 54, 1285-1296.

King DS. 1977 - Systematics of Conidiobolus (Entomophthorales) using numerical taxonomy III. Descriptions of recognized species. Canadian Journal of Botany 55, 718-729.

Kwon-Chung KJ. 2012 - Taxonomy of fungi causing mucormycosis and entomophthoramycosis (zygomycosis) and nomenclature of the disease: Molecular mycologic perspectives. Clinical Infectious Diseases 54, S8-S15.

Liu M, Rombach MC, Humber RA, Hodge KT. 2005 - What's in a name? Aschersoniainsperata: a new pleoanamorphic fungus with characteristics of Aschersonia and Hirsutella. Mycologia 97, 249-256.

Mendoza L, Vilela R, Voelz K, Ibrahim AS, Voigt K, Lee SC. 2014 - Human fungal pathogens of Mucorales and Entomophthorales. Cold Spring Harbor Perspectives in Medicine 5(4), 1-33.

Nie Y, Yu CZ, Liu XY, Huang B. 2012 - A new species of Conidiobolus (Ancylistaceae) from Anhui, China. Mycotaxon 120, 427-435.

Nie Y, Tang XX, Liu XY, Huang B. 2016 - Conidiobolus stilbeus, a new species with mycelial strand and two types of primary conidiophores. Mycosphere 7(6), 801-809.

Posada D, Crandall KA. 1998 - MODELTEST: testing the model of DNA substitution. Bioinformatics 14, 817-818.

Ronquist F, Huelsenbeck JP. 2003 - MRBAYES 3: Bayesian phylogenetic inference under mixed models. Bioinformatics 19, 1572-1574.

Srinivasan MC, Thirumalachar MJ. 1961 - Studies on species of Conidiobolus from India - I. Sydowia, Annales Mycologici15, 237-241.

Swofford DL. 2002 - PAUP*: Phylogenetic analysis using parsimony (*and other methods), Version 4.0b10. Sinauer Associates, Sunderland.

Thompson JD, Gibson TJ, Plewniak F. 1997 - The Clustal-X windows interface: flexible strategies for multiple sequence alignment aided by quality analysis tools. Nucleic Acids Research 63, 215-228.

Vilela R, Silva SMS, Correa FR, Dominguez E, Mendoza L. 2010 - Morphologic and phylogenetic characterization of Conidiobolus lamprauges recovered from infected sheep. Journal of Clinical Microbiology 48, 427-432.

Vilgalys R, Hester M. 1990 - Rapid genetic identification and mapping of enzymatically amplified ribosomal DNA from several Cryptococcus species. Journal of Bacteriology 172, 4238-4246. 
Watanabe M, Lee K, Goto K, Kumagai S, Sugita-Konishi Y, Hara-Kudo Y. 2010 - Rapid and effective DNA extraction method with bead grinding for a large amount of fungal DNA. Journal of Food Protection 73(6), 1077-1084. 